\title{
One-year outcomes of patients with ST-segment elevation myocardial infarction during the COVID-19 pandemic
}

\author{
Kailun Phua ${ }^{1}$ - Nicholas W. S. Chew ${ }^{2} \cdot$ Vincent Sim ${ }^{3} \cdot$ Audrey A. Zhang ${ }^{2} \cdot$ Saurabh Rastogi ${ }^{4}$. \\ Pipin Kojodjojo ${ }^{4}$.Wei-Ping Daniel Chor ${ }^{5}$ Brandon Chi-Ping Koh ${ }^{5}$ Benjamin Sieu-Hon Leong ${ }^{5}$ - Zhe-Yan $\mathrm{Ng}^{2}$. \\ Benjamin Wei-Liang Tung ${ }^{2}$ - Anand Ambhore ${ }^{2,3}$. William K. F. Kong ${ }^{2,3} \cdot K^{2}$ ian-Keong Poh ${ }^{2,3}$. Ping Chai ${ }^{2,3} \cdot$ Gavin Ng $^{2,3}$. \\ Koo-Hui Chan ${ }^{2,3}$. Chi-Hang Lee ${ }^{2,3}$. Joshua Ping-Yun Loh ${ }^{2,3}$. Adrian Fatt-Hoe Low ${ }^{2,3}$. Mark Yan-Yee Chan ${ }^{2,3}$. \\ Tiong-Cheng $\mathrm{YeO}^{2,3} \cdot$ Huay-Cheem $\operatorname{Tan}^{2,3} \cdot$ Poay-Huan Loh ${ }^{2,3}$
}

Accepted: 19 August 2021 / Published online: 26 August 2021

(c) The Author(s), under exclusive licence to Springer Science+Business Media, LLC, part of Springer Nature 2021

\begin{abstract}
The pandemic has led to adverse short-term outcomes for patients with ST-segment elevation myocardial infarction (STEMI). It is unknown if this translates to poorer long-term outcomes. In Singapore, the escalation of the outbreak response on February 7, 2020 demanded adaptation of STEMI care to stringent infection control measures. A total of 321 patients presenting with STEMI and undergoing primary percutaneous coronary intervention at a tertiary hospital were enrolled and followed up over 1-year. They were allocated into three groups based on admission date-(1) Before outbreak response (BOR): December 1, 2019-February 6, 2020, (2) During outbreak response (DOR): February 7-March 31, 2020, and (3) control group: November 1-December 31, 2018. The incidence of cardiac-related mortality, cardiac-related readmissions, and recurrent coronary events were examined. Although in-hospital outcomes were worse in BOR and DOR groups compared to the control group, there were no differences in the 1-year cardiac-related mortality (BOR 8.7\%, DOR 7.1\%, control 4.8\%, $\mathrm{p}=0.563$ ), cardiac-related readmissions (BOR $15.1 \%$, DOR $11.6 \%$, control $12.0 \%, \mathrm{p}=0.693$ ), and recurrent coronary events (BOR 3.2\%, DOR 1.8\%, control 1.2\%, $\mathrm{p}=0.596$ ). There were higher rates of additional PCI during the index admission in DOR, compared to BOR and control groups $(\mathrm{p}=0.027)$. While patients admitted for STEMI during the pandemic may have poorer in-hospital outcomes, their long-term outcomes remain comparable to the pre-pandemic era.
\end{abstract}

Keywords COVID-19 $\cdot$ ST-elevation myocardial infarction $\cdot$ Primary percutaneous coronary intervention

\section{Highlights}

Kailun Phua, NicholasW.S. Chew and Vincent Sim contributed equally to the manuscript

\section{Poay-Huan Loh}

poay_huan_loh@nuhs.edu.sg

1 Department of Medicine, National University Health System, Singapore, Singapore

2 Department of Cardiology, National University Heart Center, National University Health System, Singapore, Singapore

3 Yong Loo Lin School of Medicine, National University of Singapore, Singapore, Singapore

4 Department of Cardiology, Ng Teng Fong General Hospital, Singapore, Singapore

5 Emergency Medicine Department, National University Hospital, National University Health System, Singapore, Singapore
- Short-term STEMI outcomes during the COVID-19 pandemic are poorer

- However, 1-year outcomes can remain similar to the prepandemic period

- Conducting staged PCIs during the index admission reduces potential exposure

- Increased telemedicine consult allows for closer followup

- These strategies help to maintain the standard of postSTEMI care 


\section{Introduction}

Studies have shown that the Coronavirus Disease-2019 (COVID-19) pandemic has led to a significant decline in ST-segment elevation myocardial infarction (STEMI) admissions globally $[1,2]$. Besides, the overall door-toballoon time (D2B) has been significantly prolonged during the pandemic. Moreover, there were observations that patients with STEMI admitted during the pandemic had more adverse outcomes than the pre-pandemic period, such as higher serum troponin levels [3], decreased left ventricular ejection fraction (LVEF) [4], increased coronary thrombotic burden [5], and higher in-hospital complications [6]. Nevertheless, on a global scale, in-hospital mortality during the pandemic was not significantly different from that of the period before the pandemic. However, the long-term outcome of STEMI patients admitted during the pandemic remains unknown at this stage and concerns raised on the long-term sequalae of such patients are not unfounded.

As of March 31 2021, Singapore has experienced one of the highest volumes of COVID-19 cases with 60,381 cases $(10,261 / 1,000,000$ population) and 30 deaths [7, 8]. In response to the initial surge, health authorities raised the Disease Outbreak Response System Condition (DORSCON) alert level to Orange, the second highest level, on 7 February 2020. This necessitated urgent infection control and public health measures such as deferring all elective procedures. The short-term outcomes of patients admitted with STEMI during the pandemic in Singapore were similar to the trends observed throughout the globe, with an initial delay in D2B time, and more complications such as out-of-hospital cardiac arrest, intubation, acute mitral regurgitation. However, similar in-hospital mortality rates were observed when comparing those admitted during and before the pandemic [9]. With overall worsened STEMI care metrics during the pandemic, there are concerns that the long-term outcomes of patients presented during the pandemic could be adversely affected. To the best of our knowledge, this study is the first to examine the 1-year clinical outcomes of such STEMI patients.

\section{Methods}

This is a retrospective single-centre cohort study of patients who presented to a major percutaneous coronary intervention (PCI) capable hospital in Singapore, with STEMI and underwent primary PCI. This hospital is part of the Western STEMI network, which provides
PCI services to the western region of Singapore [10]. The Western STEMI network is a hub-and-spoke system that consists of our hospital (hub) as well as two other spoke hospitals. Patients presented either via (1) direct presentation to the Emergency Department at the hub hospital, or (2) inter-hospital transfer from the two spoke hospitals. Prior to the implementation of DORSCON Orange, patients with STEMI that presented to the spoke hospitals after-hours were transferred to the hub hospital for primary PCI while the service was available in one of the spoke hospitals during office hours. However, primary PCI was fully centralised after implementation of DORSCON orange such that all STEMI patients presented to the spoke hospitals were transferred to the hub hospital for primary PCI regardless of the time of presentation so as to optimise bed occupancy and consolidate resources across the region (Supplementary Material 1). This allowed the spoke hospital to care for a large influx of suspect or confirmed COVID-19 cases. During the pandemic, the hub hospital was also actively involved in the care for COVID19 patients while resources for all acute cardiac services were maintained.

The study defined three time periods for comparison: (1) Before outbreak response (BOR) from December 1, 2019 to February 6, 2020, (2) During outbreak response (DOR) from February 7, 2020 to March 31, 2020, and (3) Control group from November 1, 2018 to December 31, 2018. This study focused on the time period before and after February 7, 2020 as this was the time when Singapore DORSCON alert level was raised to Orange, the second highest level, leading to implementation of stringent outbreak control measures and reorganization of workflow for emergency, inpatient and outpatient services. Although Singapore only reported the first confirmed COVID-19 case on January 23, 2020, the BOR period was chosen as the 'early pandemic period' since Singapore is a major international travel hub with the high possibility of earlier exposure or presence of undiagnosed and/or asymptomatic cases. A distinction between the BOR and DOR intra-pandemic periods were drawn to capture the impact of different STEMI care workflow including centralisation of primary PCI services on its outcomes. Study period for the control group in November to December 2018 was specifically chosen so that it was the closest time period to the pandemic, but its 1-year follow-up period would not be affected by the COVID-19 pandemic. Consecutive patients were enrolled according to the study time period based on the date of their index admission, and those with recurrent STEMI presented during the subsequent study periods were excluded from being enrolled in these study periods to avoid repetition. All patients were followed up for 1 year from the date of their presentation for STEMI.

STEMI was diagnosed with concomitant elevated cardiac troponins $>99$ th percentile of the upper reference limit, 
and at least one of the following: angina, electrocardiogram changes (new ST elevation in 2 contiguous leads, measuring $>0.2 \mathrm{mV}$ in leads V1-3 or $0.1 \mathrm{mV}$ in all other leads, or new onset left bundle branch block) or regional wall motion abnormality on cardiac imaging consistent with myocardial infarction [11].

Data on demographic and clinical characteristics were obtained from the ongoing hospital STEMI care database [10]. This included previous medical history, clinical status at index presentation and medications on discharge. Angiographic and procedural data including access site, culprit lesion and location, D2B time, procedural success and additional PCI were also collected from the electronic medical records.

The study defined D2B time as the time taken from the patient's arrival at the treating hospital to the time of first device deployment, defined as balloon inflation, manual thrombectomy or direct stenting, during PCI. Cardiogenic shock was defined as systolic blood pressure $<90 \mathrm{mmHg}$ for $>30 \mathrm{~min}$ or the need for inotropic support to maintain systolic blood pressure $>90 \mathrm{mmHg}$. Acute mitral regurgitation was defined as new-onset mitral regurgitation related to myocardial infarction, in comparison with the previous echocardiographic characteristics, and/or based on the physician's assessment. Being on guideline-directed medical therapy for STEMI was defined as being on dual antiplatelet therapy (aspirin and P2Y12 inhibitor), statin, beta-blocker, with the option of angiotensin-converting enzyme inhibitor or angiotensin receptor blocker if the post-STEMI LVEF was $\leq 40 \%$ or the patient had diabetes mellitus.

The primary outcome was 1 - year cardiac-related mortality. The secondary outcomes were all-cause mortality, cardiac-related readmissions and recurrent coronary events. Cardiac-related mortality was defined as any death due to any cardiovascular causes. All-cause mortality was defined as death due to any or unexplained causes. The cause of death was determined by the attending physicians. Recurrent coronary events were defined as either non-fatal myocardial infarction and/or unplanned revascularisation. Cardiac-related readmissions included combined admissions for heart failure, arrhythmia, myocardial infarction, angina and/or cardiac arrest. Other important outcomes included in-hospital mortality rates (which was defined as mortality due to any cause during the index admission), cerebrovascular events and pulmonary embolism. All 1-year outcomes included outcomes during hospitalization and follow-up period.

All statistical analyses were performed using IBM SPSS Statistics for Windows, Version 25.0. Armonk, NY. Categorical variables were described as percentages and continuous variables as mean with standard deviation (SD). Continuous variables were assessed with one-way analysis of variance (ANOVA). Categorical variables were evaluated with Pearson's chi-square test (or Fisher's Exact Test where appropriate). Kaplan-Meier curves with log-rank test were constructed to compare cardiac-related mortality, cardiacrelated readmissions and recurrent coronary events. A p-value of $<0.05$ was considered statistically significant.

\section{Results}

A total of 321 patients with STEMI underwent PCI during the study periods. There were 126 (39.2\%) STEMI patients in the BOR group, $112(34.9 \%)$ in the DOR group, and 83 $(25.9 \%)$ in the control group. There was an increase in the number of STEMI presentations from 13.0 cases/week in the BOR group, and 14.5 cases/week in the DOR group, compared to 9.5 cases/week in the control group. This trend remained unchanged for the number of STEMI presentations via direct presentation, from 8.9 cases/week in the BOR group, and 9.4 cases/week in the DOR group, compared to 6.8 cases/week in the control group. The proportion of patients who presented directly (either self-presenting or via emergency medical services) to the emergency department was less in the DOR group (65.2\%) compared to the BOR (69.0\%) and control (69.9\%) groups. Post-hoc analysis excluded 9 patients who underwent inter-hospital transfer during office hours as a result of the change in the interhospital transfer strategy during the DOR period. Despite the exclusion, the number of cases in the DOR group (13.4 cases/week in the DOR group) remained higher than the other groups. The number of STEMI patients who were treated locally in the BOR and control groups that would have been transferred if the DOR protocol was in placed would approximately be 3 patients per week. Table 1 shows the baseline demographic and clinical characteristics of the study population. Subgroup analysis of patients who presented after-hours or underwent inter-hospital transfers, showed no differences in baseline and clinical characteristics across the 3 groups. However similar trends in increased STEMI complications, such as cardiogenic shock and ischemic mitral regurgitation, were observed in the pandemic periods compared to the control period. This was summarised in Supplementary Materials 2 and 3.

During the index STEMI admission, the BOR and DOR groups had higher rates of acute mitral regurgitation and lower LVEF on discharge compared to the control group. The DOR group had significantly higher rates of sepsis and a trend towards bleeding complication rates compared to their counterparts. Compared to the control group, heart failure with Killip class 3 at presentation had an increased trend amongst patients in the BOR and DOR groups. However, the length of hospital stay was similar among the 3 groups during the index admission. Although the 
Table 1 Baseline demographics and clinical characteristics

\begin{tabular}{|c|c|c|c|c|c|}
\hline & Overall $(n=321)$ & Control $(n=83)$ & $\operatorname{BOR}(n=126)$ & $\operatorname{DOR}(\mathrm{n}=112)$ & p-value \\
\hline Age, years & $59(13)$ & $59(13)$ & $58(11)$ & $60(14)$ & 0.704 \\
\hline Gender, male & $266(82.9)$ & $70(84.3)$ & $105(83.3)$ & $91(81.3)$ & 0.839 \\
\hline Ethnicity & & & & & 0.616 \\
\hline Chinese & $166(51.7)$ & $41(49.4)$ & $65(51.6)$ & $60(53.6)$ & \\
\hline Malay & $62(19.3)$ & $21(25.3)$ & $23(18.3)$ & $18(16.1)$ & \\
\hline Indian & $70(21.8)$ & $18(21.7)$ & $28(22.2)$ & $24(21.4)$ & \\
\hline Others & $23(7.2)$ & $3(3.6)$ & $10(7.9)$ & $10(8.9)$ & \\
\hline \multicolumn{6}{|l|}{ Medical history } \\
\hline Smoking status & & & & & 0.963 \\
\hline Active smoker & $113(41.4)$ & $35(42.2)$ & $50(39.7)$ & $48(42.9)$ & \\
\hline Ex-smoker & $34(10.6)$ & $10(12.0)$ & $13(10.3)$ & $11(9.8)$ & \\
\hline Hypertension & $189(58.9)$ & $50(60.2)$ & $76(60.3)$ & $63(56.3)$ & 0.782 \\
\hline Diabetes mellitus & $135(42.1)$ & $34(41.0)$ & $58(46.0)$ & $43(38.4)$ & 0.478 \\
\hline Hyperlipidemia & $206(64.2)$ & $55(66.3)$ & $82(65.1)$ & $69(61.6)$ & 0.770 \\
\hline Previous AMI & $46(14.3)$ & $13(15.7)$ & $21(16.7)$ & $12(10.7)$ & 0.392 \\
\hline Previous PCI & $50(15.6)$ & $17(20.5)$ & $19(15.1)$ & $14(12.5)$ & 0.309 \\
\hline Previous CABG & $5(1.6)$ & $4(4.8)$ & $1(0.8)$ & 0 & 0.018 \\
\hline Previous heart failure & $11(3.4)$ & $2(2.4)$ & $7(5.6)$ & $2(1.8)$ & 0.235 \\
\hline Family history of premature CAD & $42(13.1)$ & $8(9.6)$ & $18(14.3)$ & $16(14.3)$ & 0.557 \\
\hline Stroke & $14(4.4)$ & $4(4.8)$ & $3(2.4)$ & $7(6.3)$ & 0.336 \\
\hline Chronic kidney disease & $27(8.4)$ & $6(7.2)$ & $10(7.9)$ & $11(9.8)$ & 0.788 \\
\hline Presentation & & & & & 0.739 \\
\hline Direct visit & $218(67.9)$ & $58(69.9)$ & $87(69.0)$ & $73(65.2)$ & \\
\hline Interhospital transfers & $103(32.1)$ & $25(30.1)$ & $39(31.0)$ & $39(34.8)$ & \\
\hline \multicolumn{6}{|l|}{ Index admission } \\
\hline Heart failure (Killip class 3 ) & $46(14.3)$ & $6(7.2)$ & $24(19.0)$ & $16(14.3)$ & 0.058 \\
\hline Out-of-hospital cardiac arrest & $19(5.9)$ & $6(7.2)$ & $4(3.2)$ & $9(8.0)$ & 0.239 \\
\hline Sepsis & $26(8.2)$ & $5(6.0)$ & $6(4.8)$ & $15(13.4)$ & 0.040 \\
\hline Atrial fibrillation & $19(5.9)$ & $2(2.4)$ & $8(6.3)$ & $9(8.0)$ & 0.249 \\
\hline Bleeding & $29(9.0)$ & $6(7.2)$ & $7(5.6)$ & $16(14.3)$ & 0.051 \\
\hline Requiring inotropes & 47 (14.6) & $9(10.8)$ & $22(17.5)$ & $16(14.3)$ & 0.413 \\
\hline Cardiogenic shock & $30(9.3)$ & $5(6.0)$ & $16(12.7)$ & $9(8.0)$ & 0.225 \\
\hline Acute mitral regurgitation & $81(25.2)$ & $1(1.2)$ & $39(31.0)$ & $41(36.6)$ & $<0.001$ \\
\hline Ventricular arrhythmia & $31(9.7)$ & $5(6.0)$ & $17(13.5)$ & $9(8.0)$ & 0.156 \\
\hline Stroke & $5(1.6)$ & 0 & $2(1.6)$ & $3(2.7)$ & 0.328 \\
\hline Requiring intubation & $47(14.6)$ & $8(9.6)$ & $17(13.5)$ & $22(19.6)$ & 0.133 \\
\hline Acute kidney injury & $68(21.2)$ & $23(27.7)$ & $25(19.8)$ & $30(17.9)$ & 0.224 \\
\hline LVEF on discharge & $44(14)$ & $50(9)$ & $46(11)$ & $45(10)$ & 0.004 \\
\hline Hospital stay, days & $6(8)$ & $5(4)$ & $7(12)$ & $6(6)$ & 0.411 \\
\hline In-hospital mortality & $29(9.0)$ & $4(4.8)$ & $11(8.7)$ & $14(12.5)$ & 0.179 \\
\hline \multicolumn{6}{|l|}{ Medications on discharge } \\
\hline Aspirin & $291(90.7)$ & $77(92.8)$ & $111(88.1)$ & $103(92.0)$ & 0.440 \\
\hline P2Y12 inhibitor & $314(97.8)$ & $78(94.0)$ & $124(98.4)$ & $112(100)$ & 0.015 \\
\hline Beta-blocker & $253(81.1)$ & $68(81.9)$ & $99(79.2)$ & $86(82.7)$ & 0.778 \\
\hline ACE-I/ARB & $214(68.6)$ & $55(66.3)$ & $86(68.8)$ & $73(70.2)$ & 0.846 \\
\hline Statin & 293 (91.6) & $79(95.2)$ & $112(88.9)$ & $102(91.9)$ & 0.274 \\
\hline
\end{tabular}

Categorical data presented as $\mathrm{n}(\%)$. Continuous data presented as mean values (standard deviation)

$A C E I$ angiotensin-converting enzyme inhibitor, $A M I$ acute myocardial infarction, $A R \mathrm{~B}$ angiotensin receptor blocker, $C A B G$ coronary artery bypass grafting, $C A D$ coronary artery disease, $L V E F$ left ventricular ejection fraction, $P C I$ percutaneous coronary intervention 
in-hospital mortality rate in the DOR group was almost thrice that of the control group, it was not statistically significant [BOR (8.7\%), DOR (12.5\%), control (4.8\%) groups, $\mathrm{p}=0.179$ ].

The angiographic and procedural characteristics are shown in Table 2 . The majority of patients presented with culprit lesion in the left anterior descending artery (46.1\%), followed by right coronary artery $(29.0 \%)$ and left circumflex artery $(8.1 \%)$. A lower proportion of patients had postprocedural Thrombolysis in Myocardial Infarction (TIMI) flow grading system grade 3 in the BOR group (77.0\%) followed by the DOR group (85.7\%) and control group (92.8\%), $\mathrm{p}=0.027$. Other procedural characteristics including the proportion of pre-procedural TIMI flow grade 0 , stenting and use of glycoprotein IIb/IIIa inhibitors were similar across the 3 groups. Mean D2B times were similar between the control $(74 \pm 69 \mathrm{~min})$, BOR $(67 \pm 83 \mathrm{~min})$ and DOR $(66 \pm 65 \mathrm{~min})$ groups, $p=0.764$. There was significantly higher rate of additional PCI during the index admission in the DOR group (16.1\%), followed by BOR (7.1\%) and control group (6.0\%), $\mathrm{p}=0.027$. Conversely, there was a trend towards reduced rate of additional staged $\mathrm{PCI}$ in a separate admission during the DOR group (6.3\%), compared to the BOR (11.9\%) and control groups $(13.3 \%), \mathrm{p}=0.209$.

During follow-up, the mean number of physical outpatient visits were similar across the 3 groups (BOR, 2.6 per patient; DOR, 2.3 per patient; control, 2.5 per patient; $\mathrm{p}=0.351$ ). Importantly, there was significantly higher mean number of teleconsultations in the DOR group (1.4 teleconsultations per patient), followed by BOR group ( 0.6 per patient), and control group $(0.2$ per patient, $p<0.001)$. On discharge, there were fewer patients on guideline-directed medical therapy in the DOR group $(70.5 \%)$, followed by the BOR $(80.2 \%)$ and the control groups $(89.2 \%, \mathrm{p}=0.006)$. On follow-up, there was improvement in the proportion of patients being on guideline-directed medical therapy in the DOR group (85.7\%), followed by the BOR group (84.9\%), compared to the control group $(78.3 \%), p=0.332$.

The 1-year cardiac-related mortality rates were similar among the BOR (8.7\%), DOR (7.1\%) and control groups $(4.8 \%, \mathrm{p}=0.563)$. There was a trend towards increased heart failure readmissions in the BOR group (7.9\%), followed by

Table 2 Angiographic and procedural characteristics

\begin{tabular}{|c|c|c|c|c|c|}
\hline & Overall $(\mathrm{n}=321)$ & Control $(\mathrm{n}=83)$ & $\mathrm{BOR}(\mathrm{n}=126)$ & $\operatorname{DOR}(\mathrm{n}=112)$ & p-value \\
\hline Radial access & $225(70.1)$ & $55(66.3)$ & $90(71.4)$ & $80(71.4)$ & 0.676 \\
\hline Culprit vessel & & & & & 0.066 \\
\hline LM & $6(1.9)$ & $4(4.8)$ & $2(1.6)$ & 0 & \\
\hline LAD & $148(46.1)$ & $37(44.6)$ & $56(44.4)$ & $55(49.1)$ & \\
\hline Circumflex & $26(8.1)$ & $8(9.6)$ & $10(7.9)$ & $8(7.1)$ & \\
\hline RCA & $93(29.0)$ & $24(28.9)$ & $38(30.2)$ & $31(27.7)$ & \\
\hline Anterolateral branch & $10(3.1)$ & $6(7.3)$ & $2(1.5)$ & $2(1.7)$ & \\
\hline SVG & $3(0.9)$ & $2(2.4)$ & $1(0.8)$ & 0 & \\
\hline Lesion location & & & & & 0.244 \\
\hline Ostial & $9(2.8)$ & $2(2.4)$ & $3(2.4)$ & $4(3.6)$ & \\
\hline Proximal & $159(49.5)$ & $45(54.2)$ & $58(46.0)$ & $56(50.0)$ & \\
\hline Mid & $71(22.1)$ & $20(24.1)$ & $31(24.6)$ & $20(17.9)$ & \\
\hline Distal & 47 (14.6) & $14(16.9)$ & $17(13.5)$ & $16(14.3)$ & \\
\hline Multivessel disease & $189(58.9)$ & $45(54.2)$ & $78(61.9)$ & $66(58.9)$ & 0.543 \\
\hline Pre-procedural TIMI flow grade 0 & $204(65.0)$ & $57(68.7)$ & $83(65.9)$ & $64(61.0)$ & 0.301 \\
\hline Stenting & $240(74.7)$ & $61(74.4)$ & $86(68.2)$ & $83(74.1)$ & 0.837 \\
\hline Glycoprotein IIb/IIIa inhibitors & $77(24.0)$ & $15(18.1)$ & $33(26.2)$ & $29(25.9)$ & 0.341 \\
\hline Post-procedural TIMI flow grade 3 & $270(84.1)$ & $77(92.8)$ & $97(77.0)$ & $96(85.7)$ & 0.027 \\
\hline Door-to-balloon time, minutes & $69(73)$ & $74(69)$ & $67(83)$ & $66(65)$ & 0.764 \\
\hline Additional PCI & $80(24.9)$ & $18(21.7)$ & $31(24.6)$ & $31(27.7)$ & 0.494 \\
\hline During index procedure & $15(4.7)$ & $2(2.4)$ & $7(5.6)$ & $6(5.4)$ & 0.524 \\
\hline During index admission & $32(10.0)$ & $5(6.0)$ & $9(7.1)$ & $18(16.1)$ & 0.027 \\
\hline Staged admission & $33(10.3)$ & $11(13.3)$ & 15 (11.9) & $7(6.2)$ & 0.209 \\
\hline Duration between PCI, days & $3(13)$ & $5(21)$ & $3(10)$ & $2(7)$ & 0.289 \\
\hline
\end{tabular}

Categorical data presented as $\mathrm{n}(\%)$. Continuous data presented as mean values (standard deviation)

$L A D$ left anterior descending artery, $L M$ left main coronary artery, $P C I$ percutaneous coronary intervention, $R C A$ right coronary artery, $S V G$ saphenous vein graft, TIMI thrombolysis in myocardial infarction flow grading system 
Table 3 One-year study outcomes

\begin{tabular}{|c|c|c|c|c|c|}
\hline & Overall $(n=321)$ & Control $(n=83)$ & $\operatorname{BOR}(n=126)$ & $\operatorname{DOR}(\mathrm{n}=112)$ & p-value \\
\hline All-cause mortality & $38(11.8)$ & $6(7.2)$ & $18(14.3)$ & $14(12.5)$ & 0.292 \\
\hline Cardiac-related mortality & $23(7.2)$ & $4(4.8)$ & $11(8.7)$ & $8(7.1)$ & 0.563 \\
\hline Recurrent coronary event & $7(2.2)$ & $1(1.2)$ & $4(3.2)$ & $2(1.8)$ & 0.596 \\
\hline Non-fatal MI & $6(1.9)$ & $1(1.2)$ & $3(2.4)$ & $2(1.8)$ & 0.821 \\
\hline Unplanned revascularization & $4(1.2)$ & $1(1.2)$ & $2(1.6)$ & $1(0.9)$ & 0.890 \\
\hline Cardiac-related readmission & $42(13.1)$ & $10(12.0)$ & $19(15.1)$ & $13(11.6)$ & 0.693 \\
\hline MI & $6(1.9)$ & $1(1.2)$ & $3(2.4)$ & $2(1.8)$ & 0.825 \\
\hline Heart failure & $18(5.6)$ & $1(1.2)$ & $10(7.9)$ & $7(6.3)$ & 0.110 \\
\hline Arrhythmia & $3(0.9)$ & 0 & $1(0.8)$ & $2(1.8)$ & 0.430 \\
\hline Angina & $7(2.2)$ & $2(2.4)$ & $3(2.4)$ & $2(1.8)$ & 0.939 \\
\hline Cardiac arrest & $2(0.6)$ & 0 & $1(0.8)$ & $1(0.9)$ & 0.701 \\
\hline \multicolumn{6}{|l|}{ Other complications } \\
\hline Pulmonary embolism & $2(0.6)$ & $1(1.2)$ & $1(0.8)$ & 0 & 0.545 \\
\hline Cerebrovascular events & $9(2.8)$ & $3(3.6)$ & $2(1.6)$ & $4(3.6)$ & 0.569 \\
\hline
\end{tabular}

Categorical data presented as $\mathrm{n}(\%)$

$M I$ myocardial infarction

the DOR (6.3\%) and the control group (1.2\%), $\mathrm{p}=0.110$. Overall cardiac-related readmission and recurrent coronary events were similar across the three groups. There were no differences in pulmonary embolism and cerebrovascular event rates across the study groups (Table 3 ).

Kaplan-Meier curves demonstrated similar cardiac readmissions (log-rank $=0.443, p=0.801)$, recurrent
Fig. 1 Kaplan-Meier curves of cardiac-related readmissions in STEMI patients according to the three study periods

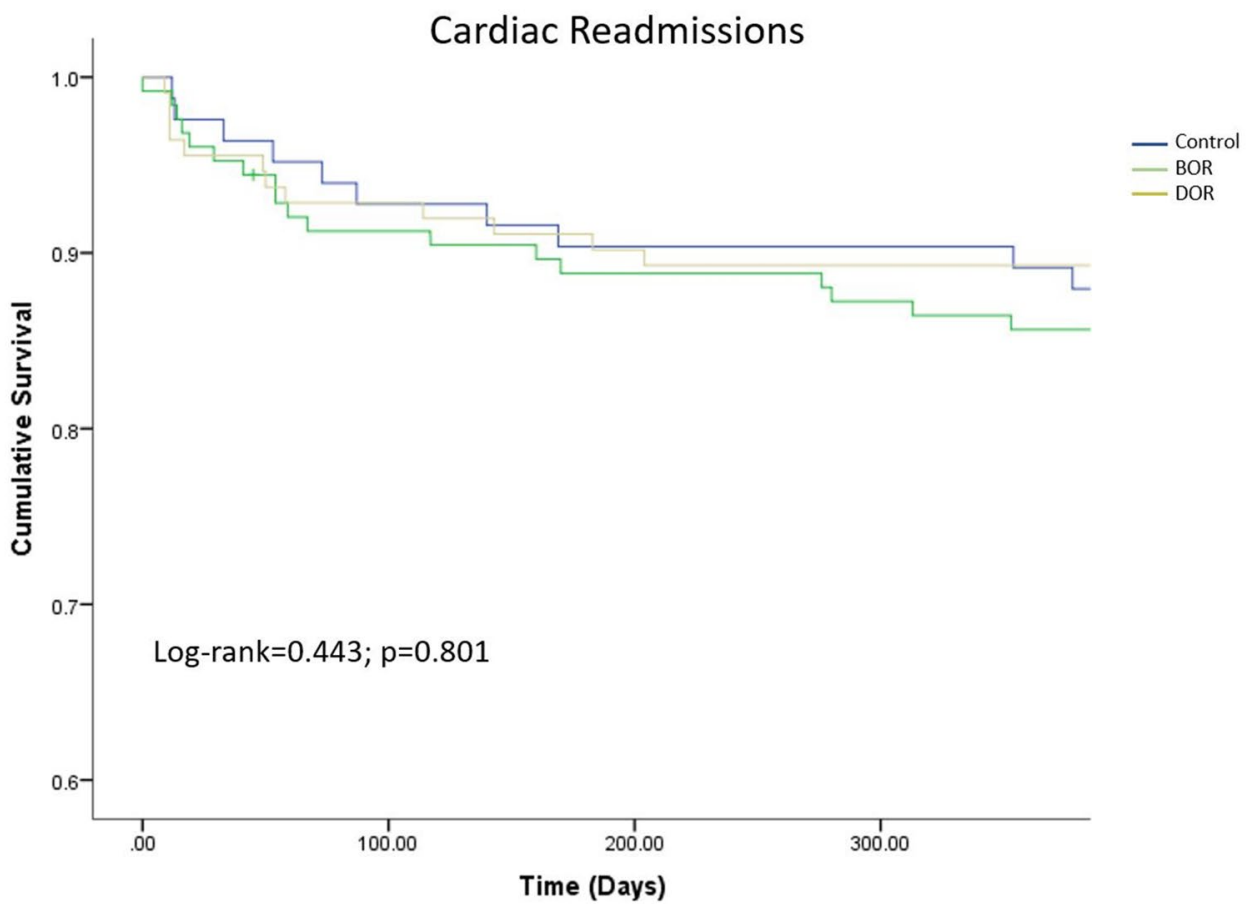

Number at risk

\begin{tabular}{l|c|c|c|c}
\hline & Baseline & 4 months & $\mathbf{8}$ months & 12 months \\
\hline Control & 83 & 76 & 74 & 72 \\
\hline BOR & 126 & 112 & 110 & 106 \\
\hline DOR & 112 & 102 & 99 & 99 \\
\hline
\end{tabular}


Fig. 2 Kaplan-Meier curves of total recurrent coronary events in STEMI patients according to the three study periods

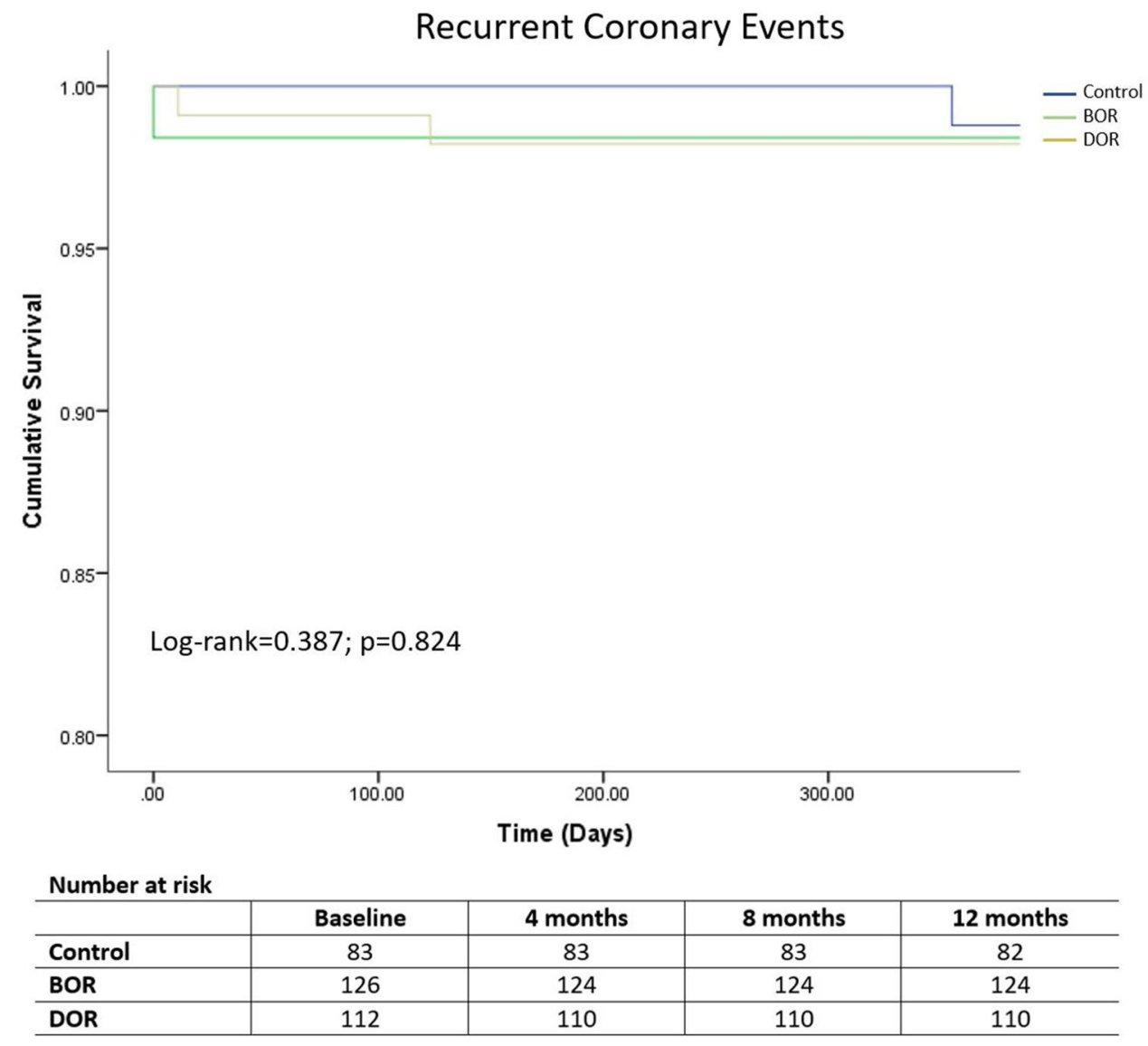

coronary events $(\log -\mathrm{rank}=0.387, \mathrm{p}=0.824)$ and cardiacrelated mortality (log-rank $=1.331, \mathrm{p}=0.514)$ across the three groups over 1 year (Figs. 1, 2, 3).

\section{Discussion}

To our knowledge, this study is the first to examine the 1-year outcomes of patients with STEMI undergoing primary PCI during the COVID-19 pandemic. The study demonstrated an increase in overall STEMI presentations in the BOR and DOR period, with worse in-hospital STEMI outcomes such as higher rates of acute mitral regurgitation, lower proportion of patients achieving post-PCI TIMI 3 flow and lower LVEF on discharge, as compared to the control group. However, there was no difference in the 1-year cardiac-related readmission rates among the three groups, although there was a trend towards an increased heart failure readmission in the BOR and DOR groups compared to the control group. Despite the higher in-hospital complication rates, in-hospital mortality remained similar across all groups and this trend of mortality and recurrent coronary event rates was sustained over 1 year. Compared to the BOR and control periods, additional PCI rate during the index admission over the DOR period was significantly higher with a consequent reciprocal trend towards lower incidence of staged PCI in a separate admission.

Studies from the Western countries have reported reduction in the incidence of STEMI admissions during the pandemic [12, 13]. On the contrary, our hospital experienced an increase in STEMI cases before and during the outbreak response to COVID-19 pandemic, compared to the nonpandemic period. This could partly be the result of strategy adopted by our regional STEMI network to centralise primary PCI service at our hospital. This strategy took advantage of the geographical proximity of healthcare hospitals within the West of Singapore (approximately $3.1 \mathrm{~km}$ and $7.3 \mathrm{~km}$ distance between hub hospital and the other two spoke hospitals) which allowed timely inter-hospital transfers. Although there was an increase in percentage of STEMI patients presenting as inter-hospital transfers in the DOR group (34.8\%), compared to the BOR $(31.0 \%)$ and control groups $(30.1 \%)$, the absolute number of interhospital transfers were the same between the pandemic periods $(n=39)$. Hence, the impact of the change in interhospital transfer strategy during the DOR period might not be significant. Our previous study demonstrated no significant D2B delay in inter-hospital transfers between the BOR and DOR periods, with majority of patients 
Fig. 3 Kaplan-Meier curves of cardiac-related mortality in STEMI patients according to the three study periods

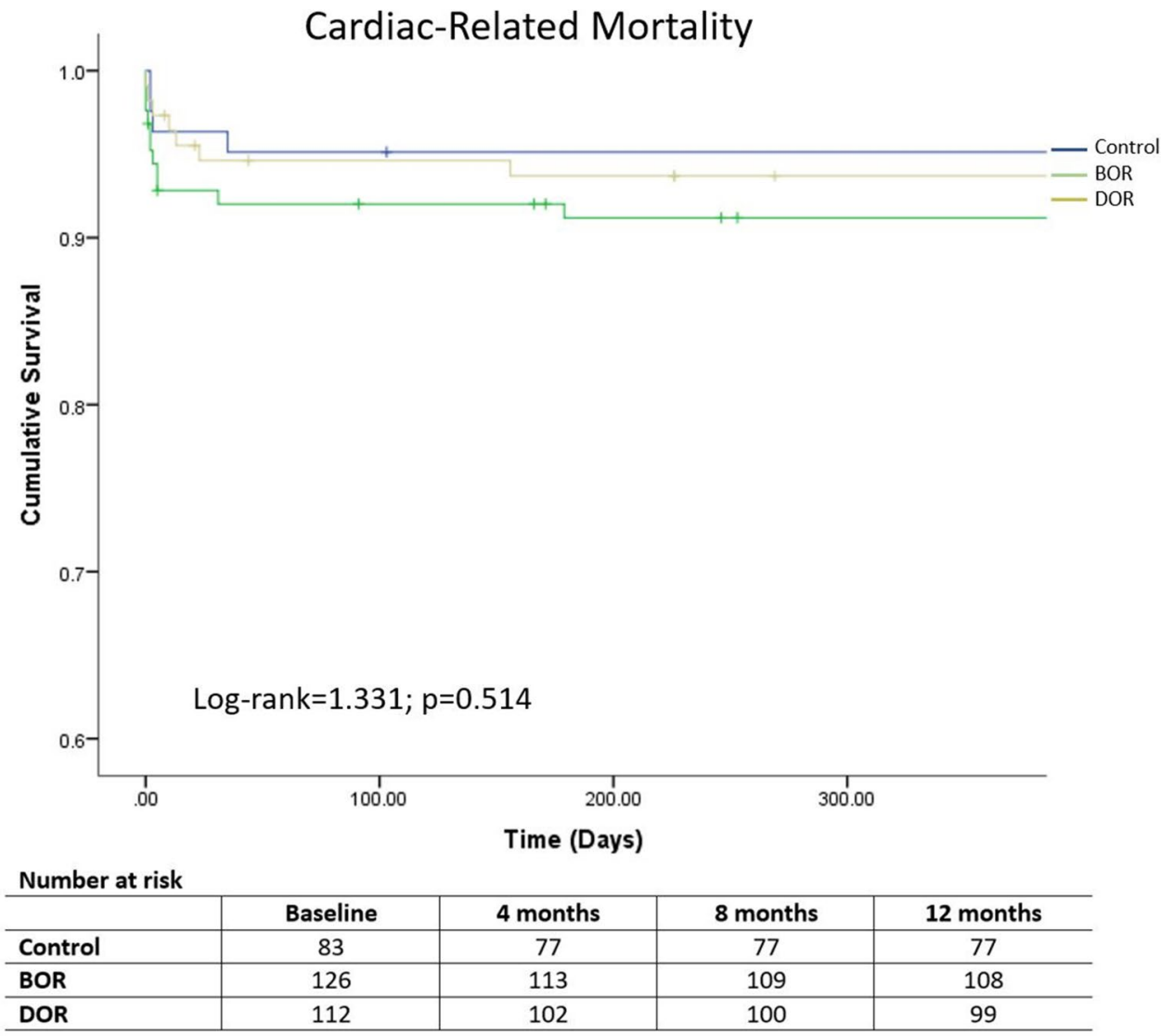

treated within the international standard of D2B time of less than 120 min [9]. This allowed the other hospitals to divert resources in providing care for suspected or confirmed COVID-19 cases. However, our observation could also reflect an actual increase in the incidence of STEMI within our region not related to the pandemic. Importantly, the number of STEMI cases were similar between the periods before and during implementation of disease outbreak response indicating that the overall incidence of STEMI during the pandemic within our region did not reduce as what was experienced by the Western countries.

A recent meta-analysis on the global impact of the pandemic on patients presenting with STEMI demonstrated no significant differences in in-hospital mortality among STEMI patients admitted during and before the pandemic [1]. However, multiple investigators have reported prolonged systemic delays during the pandemic with significantly longer symptom-to-first medical contact time and prolonged D2B time [3, 14-18]. These led to concerns that such late STEMI presentation and prolonged total ischemic time would ultimately cause adverse clinical outcomes [19]. Our study found that patients tended to present in more severely ill states with a higher proportion of Killip class 3 heart failure, acute mitral regurgitation, sepsis, and bleeding in the BOR and DOR groups.

The BOR group was similar to the DOR group in both the in-hospital and long-term outcomes despite the stringent infectious control measures and dynamic changes in STEMI care processes during the DOR period. The global trend suggests that prolonged symptom-to-first medical contact time and delayed door-to-balloon time [20] due to the reluctance in seeking medical care [21] or fear of COVID-19 transmission in the hospital [9, 22] might explain this similarity between the BOR and DOR groups. It seems that the overall public perception and initial frontline delays might have led to patients presenting in more severe conditions [4]. Compared to the control, those admitted during the pandemic also had poorer left ventricular function and a higher coronary thrombotic burden with lower proportion of patients had post-procedural TIMI flow grade 3 . These findings are consistent with those observed in other studies across the world and could occur even in regions with a relatively low prevalence of COVID-19 [3, 4, 22-24]. Notably, with the change in STEMI care processes during the DOR period to cope with the increased demand on the hospital system, there was increased rate of additional PCI during the 
index admission in order to reduce readmission rate for staged PCI. Despite the prolonged total ischemic time and adverse short-term STEMI outcomes, long-term outcomes were similar across all groups.

Studies have demonstrated that patients with cardiogenic shock have higher 30-day mortality, but those who survived the initial phase of AMI tend to have favourable long-term clinical outcomes [25]. Despite a worse clinical state at presentation among the BOR and DOR patients, our study demonstrated relatively low all-cause mortality rates in all the patients who survived the index admission (control: $2.4 \%$ vs BOR: $5.5 \%$ vs DOR: 0\%), suggesting that STEMI patients have good 1-year survival. However, the risk of heart failure might only manifest itself after a longer period of time. The poorer post-MI metrics during the pandemic among our patients have led to a trend towards higher readmission rates for heart failure in the pandemic groups. Therefore, longerterm follow-up of these patients is warranted despite the relatively reassuring 1-year mortality and cardiac-related readmission rate.

Intensive guideline directed therapy and closer monitoring are warranted for these patients with reduced LVEF following STEMI. Unique challenges arise in managing these patients during the pandemic with an increased focus on telemedicine as an alternative avenue to reduce hospital visits, whilst concurrently managing these patients adequately by providing medical education and optimising guideline directed medical therapy. Telemedicine consultations were offered to the patients according to our hospital protocol. This involved telephone calls or video conferencing led by both physicians and nurse practitioners at set intervals after discharge. These consultations would focus on patient symptoms, concerns, medication compliance, as well as remote blood tests and blood pressure monitoring. Changes to the medications could also be made via teleconsultation should there be a need for medication dosage adjustment [26]. Medication delivery service fully subsidised by the health authority was also provided during the pandemic.

Although there was an increase in the use of telemedicine during the pandemic, the proportion of patients on guideline directed medical therapy in accordance with international standards [26] remained satisfactory. Our preliminary findings are encouraging such that telemedicine did not seem to compromise the care of these patients; in fact, the 1-year cardiac-related readmission, recurrent coronary events and mortality rates did not differ across the study periods. This suggests that reasonable standard of care could be sustained in the medium term. A recent trial has demonstrated the feasibility and safety of nurse practitioner-led telemedicine in a low-risk post-myocardial infarction population with no differences in safety events or target medication doses when compared to standard outpatient care [27]. However, further

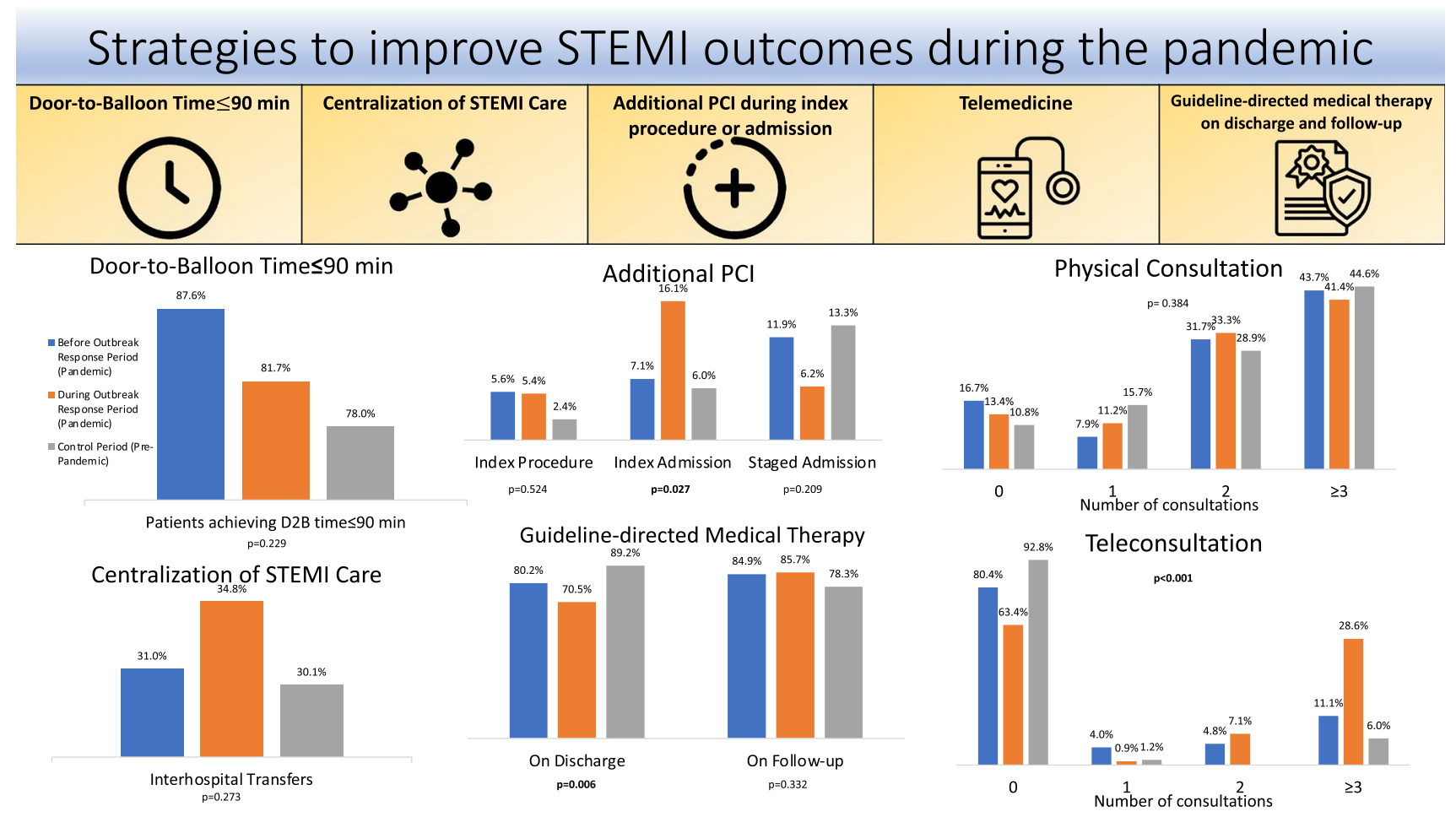

Fig. 4 Strategies to improve STEMI outcomes during the pandemic. D2B door-to-balloon, PCI percutaneous coronary intervention, STEMI STsegment elevation myocardial infarction 
studies are needed to evaluate the role of telemedicine in STEMI patients during the pandemic.

Several strategies implemented by our hospital might have contributed to the overall favorable long-term outcomes of STEMI patients admitted during the pandemic (Fig. 4). These include the centralization of acute STEMI care and increase in telemedicine consultations for subsequent follow-up with satisfactory adherence to guidelinedirected medical therapy during the pandemic period. We adopted a modified revascularization strategy in performing elective staged PCI to obstructive non-culprit lesions during the index admission, to alleviate the need for subsequent planned readmissions for staged PCI, and therefore relieving additional burden on the already strained healthcare system. This is in line with current international recommendations and could reduce the potential exposure of catherization laboratory staffs to COVID-19 during a staged procedure [28]. In addition, despite the high number of COVID-19 cases during the BOR period, our hospital took the stance to maintain all acute cardiac services including primary PCI service with full complement of staffs and only few were re-assigned to duties related to COVID-19 patient care and outbreak management strategies.

\section{Limitations}

Our study has several limitations that merit consideration. Firstly, it is a single-center retrospective observational study with a small sample size. Nevertheless, our analysis was based on consecutive patients enrolled in our STEMI database, and it reflects the actual treatment processes of a STEMI network during the pandemic. Secondly, our control period might not be representative of our current practice but it was the most recent period possible during which the 1-year follow-up would not be affected by the pandemic. Thirdly, even though there was no difference in the 1-year mortality across the study groups, we could not exclude that there might be unaccounted pre-hospital deaths. However, this is similar to most national and international STEMI registries in which only patients present to cardiac catherization laboratory are enrolled. Finally, we did not investigate the outcome of patients who presented with coronary events on the remaining spectrum of acute coronary syndrome, namely non-STEMI and unstable angina. Further research involving these patients could provide a complete understanding of overall impact of the pandemic on the care of patients with acute coronary syndrome and their outcomes. Lessons learnt from such studies could potentially help the improvement and planning of acute coronary care services during current and future infectious disease outbreaks.

\section{Conclusion}

Despite an overall worse in-hospital outcomes, the 1-year cardiac-related readmissions, recurrent coronary events, and cardiac-related mortality among patients presenting with STEMI during COVID-19 pandemic did not worsen.

Supplementary Information The online version contains supplementary material available at https://doi.org/10.1007/s11239-021-02557-6.

Acknowledgements We wish to express our deepest gratitude to colleagues who put themselves in the frontline and cared for these patients.

Funding No funds, grants or other support was received.

Data availability Not applicable.

Code availability Not applicable.

\section{Declarations}

Conflict of interest The authors have no relevant financial or non-financial interests to disclose.

Ethical approval This study was conducted in accordance to the revised Declaration of Helsinki and approved by the institutional and local ethics committee (NHG DSRB No. 2013/00442).

Informed consent Informed consent was obtained from all participants included in the study where possible.

\section{References}

1. Rattka M, Dreyhaupt J, Winsauer C et al (2021) Effect of the COVID-19 pandemic on mortality of patients with STEMI: a systematic review and meta-analysis. Heart 107:482-487. https://doi. org/10.1136/heartjnl-2020-318360

2. Han Y (2020) A treatment strategy for acute myocardial infarction and personal protection for medical staff during the COVID-19 epidemic: the Chinese experience. Eur Heart J 41:2148-2149. https://doi.org/10.1093/eurheartj/ehaa358

3. Gramegna M, Baldetti L, Beneduce A et al (2020) ST-segmentelevation myocardial infarction during COVID-19 pandemic: insights from a regional public service healthcare hub. Circ Cardiovasc Interv 13(8):e009413. https://doi.org/10.1161/CIRCI NTERVENTIONS.120.009413

4. Wilson SJ, Connolly MJ, Elghamry Z et al (2020) Effect of the COVID-19 pandemic on ST-segment-elevation myocardial infarction presentations and in-hospital outcomes. Circ Cardiovasc Interv 13:e009438. https://doi.org/10.1161/CIRCINTERVENTIO NS. 120.009438

5. Reinstadler SJ, Reindl M, Lechner I et al (2020) Effect of the COVID-19 pandemic on treatment delays in patients with 
ST-segment elevation myocardial infarction. J Clin Med 9(e2183):2183. https://doi.org/10.3390/jcm9072183

6. De Rosa S, Spaccarotella C, Basso C et al (2020) Reduction of hospitalizations for myocardial infarction in Italy in the COVID19 era. Eur Heart J 41:2083-2088. https://doi.org/10.1093/eurhe artj/ehaa409

7. Ministry of Health, Singapore. "COVID-19 situation report." https://covidsitrep.moh.gov.sg/. Accessed 31 Mar 2021

8. Li TYW, Ngiam JN, Chew NWS et al (2021) Low incidence of cardiac complications from COVID-19 and its treatment among hospitalised patients in Singapore. Ann Acad Med Singap 50(6):490-493. https://doi.org/10.47102/annals-acadmedsg. 202122

9. Chew NWS, Sia CH, Wee HL et al (2020) Impact of the COVID19 pandemic on door-to-balloon time for primary percutaneous coronary intervention. Circ J 85(2):139-149. https://doi.org/10. 1253/circj.CJ-20-0800

10. Tung BWL, Ng ZY, Kristanto W et al (2021) Characteristics and outcomes of young patients with ST segment elevation myocardial infarction undergoing primary percutaneous coronary intervention: retrospective analysis in a multiethnic Asian population. Open Heart 8(1):e001437. https://doi.org/10.1136/openh rt-2020-001437

11. Hartikainen TS, Sörensen NA, Haller PM et al (2020) Clinical application of the 4th universal definition of myocardial infarction. Eur Heart J 41:2209-2216. https://doi.org/10.1093/eurheartj/ ehaa035

12. De Filippo O, D’Ascenzo F, Angelini F et al (2020) Reduced rate of hospital admissions for ACS during Covid-19 outbreak in Northern Italy. N Engl J Med 383(1):88-89. https://doi.org/10. 1056/NEJMc2009166

13. Zaleski AL, Taylor BA, McKay RG, Thompson PD (2020) Declines in acute cardiovascular emergencies during the COVID19 pandemic. Am J Cardiol 129:124-125. https://doi.org/10. 1016/j.amjcard.2020.05.029

14. Abdelaziz HK, Abdelrahman A, Nabi A et al (2020) Impact of COVID-19 pandemic on patients with ST-segment elevation myocardial infarction: insights from a British cardiac center. Am Heart J 226:45-48. https://doi.org/10.1016/j.ahj.2020.04.022

15. Xiang D, Xiang X, Zhang W et al (2020) Management and outcomes of patients with STEMI during the COVID-19 pandemic in China. J Am Coll Cardiol 76(11):1318-1324. https://doi.org/ 10.1016/j.jacc.2020.06.039

16. Erol MK, Kayıkçıŏ̆lu M, Kılıçkap M et al (2020) Treatment delays and in-hospital outcomes in acute myocardial infarction during the COVID-19 pandemic: a nationwide study. Anatol J Cardiol 24(5):334-342. https://doi.org/10.14744/AnatolJCardiol. 2020.98607

17. Fileti L, Vecchio S, Moretti $C$ et al (2020) Impact of the COVID19 pandemic on coronary invasive procedures at two Italian high-volume referral centers. J Cardiovasc Med 21(11):869-873. https://doi.org/10.2459/JCM.0000000000001101

18. Tomasoni D, Adamo M, Italia L et al (2020) Impact of COVID2019 outbreak on prevalence, clinical presentation and outcomes of ST-elevation myocardial infarction. J Cardiovasc Med 21(11):874-881. https://doi.org/10.2459/JCM.0000000000 001098
19. Nan J, Jia R, Meng S (2021) The impact of the COVID-19 pandemic and the importance of telemedicine in managing acute ST segment elevation myocardial infarction patients: preliminary experience and literature review. J Med Syst 45(1):9. https://doi. org/10.1007/s10916-020-01703-6

20. Chew NWS, Ow ZGW, Teo VXY et al (2021) The global impact of the COVID-19 pandemic on STEMI care: a systematic review and meta-analysis. Can J Cardiol. https://doi.org/10.1016/j.cjca. 2021.04.003

21. Claeys MJ, Argacha J-F, Collart P et al (2020) Impact of COVID19-related public containment measures on the ST elevation myocardial infarction epidemic in Belgium: a nationwide, serial, cross-sectional study. Acta Cardiol. https://doi.org/10.1080/00015 385.2020.1796035

22. Hammad TA, Parikh M, Tashtish N, Lowry CM, Gorbey D, Forouzandeh $\mathrm{F}$ et al (2020) Impact of COVID-19 pandemic on ST-elevation myocardial infarction in a non-COVID-19 epicenter. Catheter Cardiovasc Interv. https://doi.org/10.1002/ccd.28997.10. 1002/ccd.28997

23. Cammalleri V, Muscoli S, Benedetto D et al (2020) Who has seen patients with ST-segment-elevation myocardial infarction? First results from Italian real-world coronavirus disease 2019. J Am Heart Assoc 9(19):e017126. https://doi.org/10.1161/JAHA.120. 017126

24. Little CD, Kotecha T, Candilio L et al (2020) COVID-19 pandemic and STEMI: pathway activation and outcomes from the pan-London heart attack group. Open Heart 7(2):e001432. https:// doi.org/10.1136/openhrt-2020-001432

25. Thiele H, Akin I, Sandri M et al (2018) One-year outcomes after PCI strategies in cardiogenic shock. N Engl J Med 379(18):1699_ 1710. https://doi.org/10.1056/NEJMoa1808788

26. Ibanez B, James S, Agewall S, ESC Scientific Document Group et al (2018) 2017 ESC guidelines for the management of acute myocardial infarction in patients presenting with ST-segment elevation. Eur Heart J 39(2):119-177. https://doi.org/10.1093/ eurheartj/ehx393

27. Chan MY, Koh KWL, Poh SC, IMMACULATE Investigators et al (2020) Remote postdischarge treatment of patients with acute myocardial infarction by allied health care practitioners vs standard care: the IMMACULATE randomized clinical trial. JAMA Cardiol. https://doi.org/10.1001/jamacardio.2020.6721

28. Mahmud E, Dauerman HL, Welt FGP et al (2020) Management of acute myocardial infarction during the COVID-19 pandemic: a position statement from the society for cardiovascular angiography and interventions (SCAI), the American College of Cardiology (ACC), and the American College of Emergency Physicians (ACEP). J Am Coll Cardiol 76(11):1375-1384. https://doi.org/10. 1016/j.jacc.2020.04.039

Publisher's Note Springer Nature remains neutral with regard to jurisdictional claims in published maps and institutional affiliations. 